\title{
DESAFIOS ÉTICOS EN LAS AULAS UNIVERSITARIAS
}

\section{Aprendizajes docentes}

José Juncosa Blasco ${ }^{1}$

Director de la Carrera de Antropología Aplicada de la Universidad Politécnica Salesiana

\section{Introducción}

Cuando me solicitaron este artículo para la revista Sophia decidí dirigirme a los lectores en primera persona porque es la mejor manera para cumplir el propósito de este escrito: recuperar y reflexionar la práctica docente a partir de los desafíos y cuestionamientos éticos y morales que surgen a diario del contacto con estudiantes y colegas. No se trata, entonces, de desarrollar una determinada faceta de la deontología o ética docente sino de recuperar narrativamente cómo mi práctica docente cotidiana, la de un profesor de antropología, me ha involucrado cada vez más en la búsqueda de respuestas y marcos referenciales más profundos y definitivos relacionados con la ética y la moral.

La experiencia narrada -que puede ser la de cualquiera de ustedes- de seguro no tendrá nada de significativo y especialmente nada de relevante, salvo que contribuya a que los colegas reconozcan sus propios pasos e incorporen la reflexión ética en la docencia, un desafío no sólo inevitable sino también necesario. 
Admito no haber encontrado respuestas para cada pregunta ni soluciones acabadas para cada una de las cuestiones aquí planteadas. De verdad, eso me tranquiliza, pues al fin y al cabo docentes y estudiantes somos semejantes porque estamos hechos de la misma madera, de aquella en que las hendiduras de las dudas e interrogantes irresueltas son más numerosas que las certezas. Sin renunciar a un horizonte de verdad, la actitud que prima consiste en no esquivar los desafíos con recetas y salidas fáciles engañosamente seguras y confiables. Más bien, les he dado el valor de hallazgos provisionales que anuncian otros más profundos y definitivos pero aún pendientes por identificar.

Les propongo el siguiente itinerario: desarrollaré en primer lugar una brevísima aproximación a los conceptos básicos que, aunque incompleta, proporciona un mínimo acuerdo en el significado de los términos. En segundo lugar, expondré una serie de aprendizajes (no enseñanzas) surgidos de la práctica docente, de las conversaciones y de los debates informales con estudiantes y colegas. De paso, aclaro que los temas resultantes no son casuales y, aunque se vinculan más directamente con el área disciplinar de mi competencia, tocan fibras muy sensibles de la cultura contemporánea y están muy presentes en las discusiones cotidianas de todos.

He escogido tan sólo de entre otros aprendizajes las siguientes proposiciones: 1. Relacionar la moral con la política; 2. Abrazar la diversidad como valor. Estos aprendizajes tocan aspectos cruciales que pueden contribuir a ordenar y profundizar otros aspectos de la ética y de la moral.

\section{Definiciones básicas 2}

En primer lugar debemos aclarar que moral y ética no son lo mismo. En este contexto, la moral es una 
condición existencial que nos atraviesa como personas: todos inevitablemente somos morales o inmorales, pero nunca amorales porque continuamente y a cada momento debemos decidir, juzgar o actuar sobre la decisión de si obramos moralmente o no.

La ética, en cambio, es el ámbito de lo que sabemos, pensamos o discutimos sobre la moral. La Universidad es un escenario privilegiado para la ética o la reflexión teórica sobre la moral, pues en la vida cotidiana no sólo entendemos cosas diferentes cuando nos referimos a la moral; también, observamos que existe una distancia bastante grande entre las prácticas morales o inmorales y lo que sabemos de ellas; entre lo que definimos discursivamente como idealmente bueno y lo que en realidad sucede. Asimismo, descubrimos que se trata de un campo abierto a la discusión teórica y sobre el cual son mayores los desacuerdos que los acuerdos. Por tanto, este escrito forma parte de los dos ámbitos: de la moral porque recoge y se inspira en actitudes y compromisos reales; de la ética porque los convierte en objeto de reflexión y discusión teórica; es decir, iluminada por conceptos y principios abstractos.

Otro término relevante con cuyo significado debemos ponernos de acuerdo es el de los valores. Partimos afirmando que los valores no son realidades separadas: son adjetivos o aspectos que reconocemos en las cosas y en las actitudes aunque muchas veces los substantivemos. Aunque hablamos de lo bello, de lo bueno o de lo justo, en realidad reconocemos paisajes bellos, personas buenas o decisiones justas. Por lo tanto, los valores están en las cosas. Salvo la persona, no existen cosas o realidades valiosas en sí: lo son en referencia a nuestras intenciones y expectativas. Así, por ejemplo, una fotografía es valiosa sólo para mí porque me trae a la memoria un ser querido. 
Antes que entidades abstractas que se comprenden mediante los conceptos y las teorías, los valores se aprehenden con los sentimientos y los percibimos afectivamente en términos de atracción o rechazo. Los valores son más poderosos que las ideas pues lo que nos mueve a actuar no son los conceptos sino los valores. Es posible afirmar, entonces, que los valores son correlatos (correlacionados con) de los sentimientos y su percepción y evidencia va de la mano de nuestra capacidad y profundidad emocional.

No todos los valores son iguales ni pertenecen a la misma clase. Incluso se oye hablar a menudo de tablas de valores de diverso tipo. Los valores morales, sin ser los únicos, son los más importantes, pues informan e integran a todos los otros: los estéticos, los culturales e identitarios, los intelectuales, de la salud, etc. Este artículo, en consecuencia, se referirá ante todo a los valores morales.

\section{Primer aprendizaje: relacionar la moral con lo político}

La primera tentación en las aulas es reducir la ética y la discusión sobre la moral a un asunto relacionado con las actitudes privadas e individuales, no sólo ajenas a lo público y político sino en franca disonancia y contradicción con estos ámbitos. De alguna manera existe entre nosotros una cierta postura discursiva que tiende a alejar la ética tanto de la política como de la esfera pública de la vida social.

Ello puede suceder, por ejemplo, cuando se ontologizan los valores (Cfr. Ricoeur, 2007) ${ }^{3}$, es decir, cuando se los asume como realidades objetivas, inmutables y separadas de las personas y de los grupos que los encarnan, producen o interpretan. Así, quienes cultivan esta vi- 
sión suelen plantear un menú de valores plenamente identificables y susceptibles de ser clasificados, evaluados y seleccionados según su contenido individual y sin tomar en cuenta el vínculo con los grupos y las comunidades que les dan vida. La historia, en este caso, es tomada en cuenta únicamente para evidenciar la ausencia o presencia de estos valores.

Otros, según una tendencia muy importante de la reflexión contemporánea, afirman que los valores no son realidades separadas sino producidas por los grupos humanos; nos vienen dados desde fuera pero nos estructuran desde dentro sin que seamos del todo conscientes de sus contenidos o mecanismos. Los valores permiten modelar la convivencia social al convertir en emotivamente imperativa y vinculante una determinada visión o teoría de la sociedad o del mundo producida siempre por grupos sociales específicos (Cfr. Geertz, 1977). Desde esta perspectiva, lo valores permiten pasar del ser (la teoría sobre la realidad) al deber ser (las obligaciones morales que esa teoría comporta), configuran el mundo y nos proporcionan criterios de acción y de relación con los otros que aparecen como normales y naturales, sin que podamos identificar o definir con claridad cómo ello sucede y tampoco sin ser conscientes del orden social que los valores promueven. Esta visión considera que antes que las definiciones o los contenidos socialmente reconocidos son las prácticas sociales efectivamente desarrolladas por cada uno las que expresan el significado real de los valores a los que efectivamente nos adherimos.

Si partimos de la primera postura (denominada esencialista por la teoría social), la que considera a los valores como realidades objetivas y separadas, no nos queda sino asumir, reconocer o cultivar valores. Si en cambio tomamos en cuenta la segunda postura sentiremos la nece- 
sidad, primero, de nombrar e identificar nuestras prácticas para luego evaluar la teoría (el ser) y los valores (el deber ser) que ella promueve. Esta tendencia supone al menos dos cosas muy importantes para la discusión en las aulas universitarias: que la discusión sobre los valores es estéril si no se la plantea desde las teorías del mundo, de la sociedad o del individuo que los soportan; y que los valores no son comprensibles por su contenido individual sino por su inscripción en un sistema concreto de relaciones que los producen (Cfr. Bordieu, 1995).

Continuando con la primera tendencia, es posible suponer que vivimos en un mundo sin valores (la tan traída y llevada crisis de valores entendida como ausencia) y que ante tal carencia es posible adicionar, escoger y promover determinados de ellos. Para la segunda opción, en cambio, no existe la posibilidad de tal carencia pues todo orden social supone, siempre y necesariamente, valores. Para ella, no es posible una crisis de valores en el sentido de que los hemos perdido. La crisis, más bien, puede ser explicada por alguna de las siguientes razones: porque nuestra sociedad ha sustituido los valores tradicionales por otros que no logran modelar una convivencia basada en el respeto al derecho de los otros; o porque la sociedad capitalista avanzada ha perdido su capacidad para construir un horizonte ético que justifique sus acciones a pesar de saturarnos con discursos éticos altisonantes, bienpensantes y bienhablantes (Cfr. Eagleton, 2005; Cortina, 2005)4: ¿Cómo justificar la invasión a Irak a cuenta de la democracia? ¿En nombre de qué valores entender la quiebra financiera de estos últimos tiempos? ¿Cómo creer en la vida como valor supremo si los líderes mundiales que promueven su defensa desencadenan desastres globales y no dudan un momento en promover guerras fraticidas? Los valores no están ausentes; las visiones del mundo que los 
soportan y les dan fuerza y credibilidad sí parecen haberse esfumado o transformado radicalmente.

Por lo tanto, el desafío pedagógico no consiste tanto en reconvocar valores negados o inexistentes sino en evidenciar las prácticas sociales para reconstruir desde allí la teoría (el ser) y los valores reales que éstas encarnan (el deber ser). La apuesta pasa también por retomar y volver a poner en el lugar que corresponde la reflexión metafísica sobre la sociedad y la historia. Solo así el discurso sobre los valores tendrá significado, pues la discusión ética no se concentrará tanto en qué reglas respetar sino en clarificar qué pensar sobre la persona 5 .

Esta enseñanza no pretende negar la realidad de los valores, ni tampoco reducir su discusión al marco jurídico y legal de la convivencia humana. Se trata de recuperar la dimensión profundamente política de la ética en el sentido de que la moral permite cohabitar el mundo con otros. No es casual que Sócrates haya planteado la reflexión sobre las virtudes durante el surgimiento de la democracia urbana de Atenas; es decir, en un contexto en el que se requería superar el ideal heroico según el cual unos eran potenciales enemigos de otros porque se debían por entero a los conflictos y reyertas familiares. Para superar esta visión de la ética, se requería construir un lenguaje apropiado para expresar, abstractamente, los objetivos e ideales que debían ser comunes y unánimes entendidos y aplicables a todos: el bien, la verdad y la belleza. Este salto ético -y epistemológico- posibilitó el paso de una sociedad cuyo dinamismo se basaba en la conflictividad permanente de los intereses familiares y locales a otra, también conflictiva y a su manera muy excluyente pero articulada en torno al ciudadano de la polis, capaz de cultivar relaciones cooperativas y enfocadas al bien común. Sin la reflexión sobre la ética -sin el lenguaje adecuado para ex- 
presar ideales y valores comunes a todos los habitantes de la ciudad - la democracia griega hubiera sido imposible. (MacIntire, 2001: 167ss).

\section{Segundo aprendizaje: Abrazar la diversidad como valor}

La Fiesta Internacional de la Cultura y el Libro (Quito, 2008) propone con acierto y mucha inspiración el siguiente slogan: ser libres, ser diversos, ser humanos. La diversidad es un rasgo valioso (un valor) de las personas, de los grupos humanos y de la naturaleza, aún más en un mundo caracterizado por la intensidad de las relaciones y vínculos entre individuos portadores de rasgos culturales, pertenencias y múltiples identidades. No vivimos ya en una sociedad donde los extranjeros son extraños y huéspedes ocasionales a los que se les asigna un lugar. Ya no. El otro llegó para quedarse, cohabitar, compartir y reclamar los espacios. De hecho, nuestras aulas universitarias reflejan el panorama de diversidad que caracteriza las sociedades latinoamericanas en la que cohabitan personas disímiles en todos los sentidos posibles: diversas por su género, por su edad, por su credo, por sus compromisos políticos, por su identidad étnica, por sus pertenencias socioculturales, por los estilos y culturas juveniles a los que se adscriben, etc. Cada una de estas pertenencias es también un lugar de creación y recreación de valores muy diferentes. Las aulas nos recuerdan que la Universidad es también un espacio de pluridiversidad y de necesaria apertura a un proyecto social basado en la interculturalidad sin el cual sería impensable la existencia y la viabilidad de la sociedad contemporánea.

En el sentido más básico, la interculturalidad supone al menos reconocer que cada comunidad es creadora, portadora e intérprete de sus propios códigos morales 
y que ningún otro grupo tiene derecho a cuestionarlo o violentarlo. La aceptación plana de esta afirmación en apariencia descriptiva (porque no siempre somos conscientes del valor prescriptivo de tal afirmación) e incompleta (por limitarse a lo pluricultural antes que a la dimensión intercultural), políticamente correcta y ampliamente difundida en los medios no suele evidenciar el problema ético y epistémico subyacente que nos enfrenta al siguiente interrogante: ¿Cómo reconocer los valores diversos y al mismo tiempo trascender el relativismo moral? Intentaremos algunas respuestas pero afirmamos de entrada que el relativismo (un rasgo esencial de lo que algunos denominan condición posmoderna) es un momento necesario y éticamente productivo que no se debe negar sino trascender y superar transitándolo. Veamos un relato de este intento.

Una primera pista proviene de la misma metodología del trabajo de campo antropológico cuyo punto de partida consiste en considerar las prácticas sociales como legítimas y racionales desde el punto de vista del actor, constituido por sujetos reflexivos que actúan según intenciones, expectativas y metas. Este principio metodológico, ciertamente relativista, no afirma que lo racional y legítimo es al mismo tiempo moral, pero aunque lo supongamos resulta sano y moralmente productivo para el observador porque obliga a relacionar los códigos morales de los otros, por más extraños o crueles que parezcan, con la lógica de su propio mundo y a concebirlos como respuestas colectivas a problemas profundos y existenciales que pueden resultar significativas también para los desafíos irresueltos de nuestro propio grupo sociocultural. Es moralmente productivo incluso porque nos interroga acerca de la supuesta racionalidad y legitimidad que atribuimos a nuestros códigos morales a los que con frecuente y suprema irresponsabilidad solemos convertir en criterios de juicio para los valores de los otros. Tal apertura 
nos permite, incluso, usufructuar esta mirada hacia los valores de los otros porque en tal confrontación obtenemos una mejor comprensión de nuestros propios valores.

El relativismo metodológico es moralmente productivo pero, no obstante, insuficiente y comporta amenazas. Es necesario dar un segundo paso para enfrentar la posibilidad de que tal actitud metodológica modele una conciencia moral relativista que licue la importancia, gravedad y carácter necesario de nuestras propias opciones y actitudes morales, un hecho respecto al cual la práctica antropológica ha dado cuenta más de una vez. ¿Cómo podemos evitar que la diversidad del otro no implique que nos despojemos de nuestros propios valores? Asimismo, hay prácticas propias y ajenas que provocan la certeza subjetiva de que no son legítimas y pueden resultar inmorales. ¿Qué hacer y qué pensar en tales casos?

Propongo tres soluciones teóricas, de profundidad y alcance diversos, que coinciden porque combinan lo particular con lo universal sin que lo uno excluya o anule lo otro a la vez que redimensionan el relativismo para construir un posible marco común de valores; o porque, sobre todo las dos últimas propuestas reconocen en las pertenencias e identidades socioculturales y comunitarias el factor clave que nos constituye como personas al afirmar que la dimensión comunitaria es el fundamento y la fuente última de la ética. Son, por último, las comunidades morales primarias el lugar donde intentar buscar y encontrar la fuente de la moral. Ésta es la tesis central de la corriente denominada comunitarismo y que asumo plenamente como marco general de discusión ${ }^{6}$ porque nos abre a una perspectiva necesariamente plural.

La primera es la perspectiva filosófica de Levinas; la segunda, la propuesta ética denominada perspectivismo; la tercera, el concepto de posmodernidad emancipa- 
dora de Boaventura de Souza Santos. Ellas nos invitan a abrazar la diversidad sobre el transfondo de una perspectiva ética común y universal y, de seguro, superan los mínimos de la tolerancia (¿quiénes somos nosotros para atribuirnos el derecho de tolerar a otros?) y de la perspectiva multicultural (reconocemos las diferencias pero una de ellas marca la pauta y detenta la hegemonía sobre las otras). Veamos muy brevemente la tesis central de cada propuesta.

\section{a) La moralidad como horizonte anterior a la cultura se- sún la filosofía existencial de Levinas}

Una antropóloga, Nancy Scheper-Hughes (1997), se propone superar el relativismo cultural en el inicio de su estudio sobre la violencia y la vida cotidiana en un pequeño poblado de Recife (Brasil) que busca comprender la muerte prematura y no llorada de los niños muy pequeños. Su punto de partida es la recuperación de la filosofía existencial de Levinas para quien "la moralidad no pertenece a la cultura: nos permite juzgarla" (1987: 100, citado por Scheper-Hugues, 1997: 33); es decir, la ética es anterior respecto a la cultura porque todo encuentro con el otro diverso supone de por sí la existencia de una instancia previa y fundante capaz de interpelar por igual a los sujetos que se encuentran: "los antropólogos... tienen la tendencia a concebir la moralidad como algo que siempre es contingente en relación a, e inserta en, supuestos culturales específicos sobre la vida humana. Pero hay otra posición, una postura filosófica existencial, que postula lo inverso sugiriendo que la ética siempre es anterior a la cultura porque la ética presupone todo sentido y significado y por lo tanto hace que la cultura sea posible... E compromiso y la responsabilidad con el 'otro'... es 'precultural' por cuanto la existencia humana siempre presu- 
pone la presencia de otro. El que yo haya sido lanzado a la existencia humana ya presupone algo dado, una relación moral con otra (la madre) y de ella conmigo" (idem).

Esta postura pone a todas las culturas, diversidades o alteridades en un mismo plano de equidad respecto al horizonte ético previo que interpela y exige a todas por igual. La conciencia de que todos somos moralmente auditables desde este horizonte es una herramienta muy eficaz para prevenir y cuestionar el sentido de superioridad moral característico de algunos (muy pocos) militantes y comprometidos con las causas justas, arrogancia moral que alimenta, no pocas veces, la impunidad e indolencia ante casos de evidente corrupción en las agrupaciones políticas, religiosas, instituciones educativas y también en los movimientos sociales.

Levinas nos propone, por tanto, una pista muy interesante para cultivar y construir en las aulas una actitud ética verdaderamente intercultural que promueva el respeto entre las diversidades pero también el sentimiento de equidad moral, de exigibilidad y rendición de cuentas entre todas las diversidades.

\section{b) El perspectivismo ético}

El perspectivismo ético, según la ya citada Adela Cortina, propone que no existen tantos valores cuantas culturas e identidades capaz de crearlos; más bien, existe una suerte de única bolsa de valores universales respecto a los cuales cada grupo identitario es capaz de identificar, percibir y asumir tan solo algunos de ellos a partir de su propia perspectiva y sensibilidad cultural.

Se trata, entonces, de una propuesta e intento formal que busca relacionar lo universal con lo particular y cuya propuesta pedagógica consiste no tanto en promover valores sino en crear en las aulas una sensibilidad mo- 
ral, un paladar ético, capaz de reconocer y degustar no sólo los valores adscritos a la perspectiva del propio entorno cultural sino también aquellos que forman parte del ángulo de mirada de otros grupos socioculturales. En consecuencia, el reconocimiento de los otros diversos es un paso necesario para enriquecer la propia perspectiva ética y moral e incorporar aquellos valores que nuestro ángulo de mirada nos impide reconocer.

b) La propuesta ética y política de la posmodernidad emancipatoria

El conocido pensador Boaventura de Souza Santos, intelectual orgánico de los nuevos movimientos sociales y de la globalización alternativa, recupera desde otra perspectiva la diversidad más allá del relativismo. Diagnostica, en primer lugar, que la crisis contemporánea es ante todo una crisis epistémica, es decir relacionada con nuestras formas de conocer, que se irradia hacia lo ético y lo político. Por ello, una nueva ética solicita necesariamente la creación de un nuevo paradigma de pensamiento que complete el programa inconcluso de la modernidad (la solidaridad) y recupere el potencial crítico de la posmodernidad.

Nos interesa destacar de este autor las dos maneras diferentes de vivir la posmodernidad: el posmodernismo celebratorio o la radicalización de la relatividad del conocimiento y de las actitudes éticas y políticas; y el posmodernismo emancipatorio, que valora el relativismo por su capacidad crítica pero sin permitir que licue las oportunidades emancipatorias. El posmodernismo emancipatorio, al que también denomina inquietante o de oposición (De Souza Santos, 2003: 33), parte de reconocer al otro como sujeto de conocimiento y se funda en la solidaridad: "conocer es reconocer al otro como sujeto de conocimien- 
to, es progresar en el sentido de elevar al otro de estatus de objeto al estatus de sujeto. Esta forma de conocimiento como reconocimiento es la que denomino solidaridad" (Idem., 2003: 34).

La propuesta de Boaventura de Souza Santos recupera la diversidad pero la pondera a partir de su carácter emancipatorio y antihegemónico y por su capacidad de transformar el orden social; es decir, porque pueden pasar de la acción conformista a la acción rebelde: "la tarea más importante de la teoría crítica posmoderna es explorar y analizar todas aquellas formas específicas de socialización, de educación y de trabajo que promuevan la generación de subjetividades rebeldes" (Idem., 2003: 39). El cambio y la trasnformación admite múltiples futuros, no tiene una única dirección ni se fundamenta en un solo sujeto histórico (el punto crítico del marxismo).

El mensaje consiste en que no toda forma de diversidad es legítima. Algunas tienen tan solo un valor celebratorio de ratificación de la diferencia como valor en sí misma, pero no todas las diversidades conllevan la posibilidad de generar horizontes éticos viables y transformadores, capaces de articularse con otras diversidades a fin de asegurar un futuro emancipatorio. Algunas diversidades, incluso, se construyen hegemónicamente sobre la base de un programa sistemático de exclusión y negación del otro, tal como ocurre con la ideología del mestizaje (que identifica la nación con los atributos de una sola de sus identidades históricas), por ejemplo, o de la acción violenta emprendida por los skinds heads o cabezas rapadas que promueven el odio racial y la eliminación de grupos considerados impuros o ajenos a la historia y al destino prefigurado de una nación. 


\section{Conclusión}

El recorrido aquí descrito pudo haber decepcionado a más de uno porque, como se puede observar, proponemos teorías antes que consejos, soluciones o anécdotas iluminadoras. No sólo que soy consciente de este hecho; pienso además que así debe ser. Tal vez el principal aprendizaje de esta experiencia que a más de uno le pudo parecer más enredada de lo necesario, consiste en que, en las aulas universitarias todos los problemas éticos deben conducir necesariamente a la búsqueda e identificación de teorías capaces de abrir horizontes comprensivos más amplios e interpelantes. Éste es el servicio más importante que los docentes podemos ofrecer a la universidad, a nuestros alumnos y a nuestros colegas.

Y ello por una razón más profunda que las competencias y capacidades teóricas que los docentes debieran poseer para ser capaces de formular y solucionar problemas. Se trata, además, de recuperar el discurso sobre el ser, sobre la sociedad, la persona -la metafísica- como único lugar posible para construir una ética fundamentada en nuevos paradigmas de pensamiento y que nos ponga a salvo de la discusión sobre las normas y, sobre todo, de la oleada aplastante de las agendas éticas globales tan altisonantes como carentes de fuerza y credibilidad.

Por último, es posible que el ejercicio teórico no nos haga más sabios pero sin duda nos ayudará a crecer como personas morales y capaces de reflexión ética. Por lo pronto, me ha ayudado a mí mismo a incluir trabajosamente la dimensión política en las cuestiones éticas y a abrazar la diversidad como puerta de reconocimiento del otro.

Quito, noviembre del 2008. 


\section{Notas}

1 Antropólogo por la Universidad Politécnica Salesiana. Director de la Carrera de Antropología Aplicada.

2 Me remito al marco de definiciones propuesto por Adela Cortina en su libro Alianza y contrato. Política, ética y religión (Segunda Edición, Editorial Trotta, Barcelona, 2005). Una versión más breve de estos conceptos podemos hallarlos también en la misma autora: El mundo de los valores. Ética mínima y educación, Editorial El Búho, Bogotá, 2002.

3 Ricoeur afirma que todo intento de ontologizar el bien y el mal, es decir, el atribuirle una existencia real en un determinado orden cósmico o mitológico comporta la negación de la libertad humana.

4 Terry Eagleton denuncia la incapacidad del capitalismo contemporáneo para legitimar moralmente sus propias acciones porque ha renunciado a vincular el deber ser (la moral) con la reflexión sobre el ser (la metafísica), de tal manera que la moral se vuelve insostenible y vacía: "A medida que el capitalismo occidental se embarca en su etapa posmetafísica, estos códigos empiezan a perder su credibilidad. El propio clima secular y pragmático que ha creado el capitalismo les confiere el tono hueco y clerical de un sermón acerca de por qué Dios permite el genocidio. La hipocresía altisonante empieza a dejar paso al interés más explícito. Los códigos morales estrictos empiezan a relajarse a medida que las clases medias firmemente acreditadas van convirtiéndose cada vez más en algo del pasado, y a medida que la moralidad y las costumbres empiezan a reflejar un mundo bidimensional de inacción, cinismo y egoísmo. Los valores morales que reflejan lo que uno realmente hace son bastante más plausibles, pero sólo a expensas de que ya no sirvan para legitimar nuestra actividad" (2005: 160).

5 Otros autores dan cuenta de la misma hipocresía altisonante denunciada por Eagleton. Adela Cortina, por ejemplo, llama la atención sobre el desesperado intento por construir referentes éticos globalizados pero carentes de fuerza que caracteriza a no pocos organismos internacionales e incluso a la Iglesia: "Y al final los documentos episcopales no dicen nada más que lo que podría decir cualquier burócrata de la UNESCO: hay que respetar los derechos humanos, proteger el medio ambiente, evitar la xenofobia y el racismo, respetar a los ancianos y discapacitados, de los que tanto podemos aprender, y así toda esa moralina burocrática que cualquiera puede desgranar sin equivocarse un ápice, porque se ve, lee, 
oye y casi palpa en todos los discursos bienpensantes y bienhablantes. Discurso plano: sin ningún relieve. Como un electroencefalograma que refleja esa línea recta, sin altibajos, señal de una vida que se esfuma, monótona, sin fuerza vital" (2005: 180).

6 Esta es la tesis que late a lo largo de todo el trabajo de Alasdair MacIntire (2001), reforzada por otros puntos de vista provenientes de disciplinas diversas. Un ejemplo de esta perspectiva es también la propuesta lacaniana de Dufour (2001) según el cual toda persona es un sujeto simbólico; es decir, atravesado por el lenguaje y por una gramática social. Negar las pertenencias equivale a ponerse al margen de esa gramática profunda que nos constituye como personas por la cual somos capaces de escuchar al otro y podemos reclamar ser escuchados; desconocer nuestros lazos sociales significa también que hemos sucumbido a la estrategia del capitalismo que, al liberarnos de nuestras pertenencias identitarias, nos ha sometido a la servidumbre del consumo.

\section{Bibliografía}

CORTINA, Adela

2002 El mundo de los valores. Ética mínima y educación. Bogotá: Editorial El Búho.

2005 Alianza y contrato. Política, ética y religión. Barcelona: Editorial Trotta.

BORDIEU, Pierre

1995 Razones prácticas. Madrid: Anagrama.

DE SOUZA SANTOS, Boaventura de

2003 La caída del Angelus Novas. Ensayos para una nueva teoría social y una nueva práctica política. Bogotá: ILSA.

DUFOUR, Dany-Robert

2007 El arte de reducir cabezas. Sobre la servidumbre del hombre liberado en la era del capitalismo total. Paidós, Buenos Aires.

EAGLETON, Ferry

2005 Después de la teoría. Barcelona: Editorial Debate.

GEERTZ, Clifford

1997 "Ethos, visión del mundo y análisis de los símbolos sagrados". En: Cosmos, hombre y sacralidad. Lecturas dirigidas de Antropología religiosa. Segundo Moreno Yánez (comp.). Quito: Ediciones Abya Yala. 
MACINTIRE, Alasdair

2001 Tras la virtud. Barcelona: Editorial Crítica.

RICOEUR, Paul

2007 El mal. Un desafío a la filosofía y a la teología. Buenos Aires: Amorrortu Editores.

SCHEPER-HUGHES, Nancy

1997 La muerte sin llanto. Violencia y vida cotidiana en Brasil. Barcelona: Editorial Ariel. 